\title{
Motor Neurone Disease (MND) and Amyotrophic Lateral Sclerosis (ALS): Social Media Communication on Selected \#MND and \#ALS Tagged Tweets
}

\author{
Bronwyn Hemsley \\ The University of Newcastle \\ bronwyn.hemsley@newcastle.ed \\ u.au
}

\author{
Stuart Palmer \\ Deakin University \\ stuart.palmer@deakin.edu.au
}

Stephen Dann

Australian National University

stephen.dann@anu.edu.au

\author{
Wendy Goonan \\ The University of Newcastle \\ wgoonan@,bigpond.net.au
}

\begin{abstract}
In March-April 2016, 3288 original tweets tagged with \#MND \#ALS and other minor related tags (e.g., \#cureALS \#cureMND) were analysed using mixed quantitative and qualitative methods on tweet data and content. In this paper we outline the technical methods used in gathering and selecting relevant tweets for analysis, and present the results including inductive coding of content themes. The findings will inform (a) future social media research in relation to $M N D / A L S$, (b) improved supports for people with MND/ALS and their families to use Twitter for information exchange, advocacy, and as a non-verbal form of communication, and (c) knowledge for MND/ALS service providers and philanthropic organisations on ways to engage with adults with MND/ALS in Twitter.
\end{abstract}

\section{Introduction}

Twitter is as important social media platform supporting the exchange of information and interpersonal communication [1]. In bypassing speech and allowing only a short number of characters per tweet, it is a valued method of textbased communication for people with severe communication disability who cannot rely on natural speech to communicate [2,3]. In the emergent field of research on the use of social media by people with communication disability $[2,3,4,5]$ there is little attention paid to people diagnosed with Amyotrophic Lateral Sclerosis (ALS) and Motor Neurone Disease (MND), who face progressive and relentless loss of motor abilities and functions, impacting not only on their health but also on their social participation, quality of life, employment, and relationships. This group's needs are likely to differ from those of people with chronic or lifelong communication impairments, such as cerebral palsy [3]. To date, little is known about how Twitter is used by people with MND/ALS who have severe communication disability, either as a timely source of high quality health information, a method of communication, or as a form of interaction and engagement online.

In this study we utilized Twitter research methods reported previously [6] to (i) gather Twitter data using the most commonly used 'disease-related' tags of \#ALS or \#MND, and (ii) apply qualitative and quantitative analyses, including manual, inductive, and computational coding of tweet content, to identify patterns in the use of Twitter relating to $\mathrm{MND} / \mathrm{ALS}$, and content themes. The findings of this hashtag study will be presented. Implications for MND/ALS organisations using Twitter to connect with adults with MND/ALS and their families will be discussed, along with directions for future Twitter research.

\section{Background}

MND is a term used to describe a number of heterogeneous progressive neurological conditions affecting motor neurons [7]. The most common type of MND is ALS, resulting in multiple impairments and disability relating to muscle weakness, stiffness, overactive reflexes, and in some cases emotional lability [8]. Cognitive impairments occur in 20-50\% of people with MND/ALS. As the disease progresses relentlessly, MND/ALS affects the muscles used for speaking, swallowing, and breathing [9]. The cause of MND/ALS is not yet known, and there is as yet no known cure, and a median survival rate of 2-4 years [10]. MND/ALS primarily affects people aged between 43-52 years (familial cases) or aged between 58-63 years (spontaneous cases). An estimated 58\% of adults with MND/ALS are aged under 65 years of age [e.g., 11]. The loss of motor control over the 
movements for speech reduces intelligibility and, in acquiring a severe communication disability, many people with MND/ALS will need or use assistive technologies such as computer software and augmentative and alternative communication systems (e.g., communication boards, speech generating devices, mobile devices with speech output apps) to communicate $[12,13]$.

Twitter is a text-based form of interpersonal communication, Hemsley et al., [2,3] proposed that people with severe communication disability including those with MND/ALS could benefit by using Twitter to communicate and to exchange information, as Twitter supports fewer keystrokes for entering messages, does not require speech, and is tolerant of spelling errors and abbreviations that save time and effort. Furthermore, adults acquiring MND/ALS are likely to be literate and to be familiar with using the Internet and social media particularly in the age range of the majority at onset of the disease [11]. While research on specific health conditions and the use of Twitter is increasing for people with chronic or lifelong disabilities that are not lifelimiting (e.g., traumatic brain injury [4], or cerebral palsy [3]) little is known about the use of Twitter relating to people with a progressive condition MND/ALS, that gradually robs them of their speech [8], beyond its use as a useful platform for fundraising and raising awareness (e.g., the Twitter and YouTube campaign the \#ALSicebucketchallenge [14]).

\section{Method}

Ethical approval was obtained from the relevant University for harvesting of tweets tagged with \#MND \#ALS and minor related tags, and purposive sampling for potentially highly relevant tweets. The search terms '\#MND or \#ALS' were entered into the Twitter search bar daily over the period of $11^{\text {th }}$ March to $12^{\text {th }}$ April 2016 yielding 12,965 tweets. The NCapture program [15] is able to capture all publicly available data (tweets and retweets) originating directly from a specific Twitter account, as well as data arising from a search for tweets that use a particular hashtag.

All tweets were subjected to the following criteria for inclusion and exclusion in the study: (a) included: tweets in English, relating to MND/ALS; (b) excluded: duplicate or multiple batch tweets of identical wording regardless of time of sending, retweets, relating primarily to broadcasting major fundraising efforts, not in English, or identified as junk/spam. Tweets that met exclusion criteria $(n=$
$9,677)$ were removed by the first author and checked by the second author with discrepancies resolved by consensus. In total, 3,288 original tweets relating to MND/ALS without a primary focus on fundraising remained in the sample for analysis. Thus, this sample comprises a purposive and non-representative sample for the purposes of mixed methodology analytical techniques considering the use of Twitter for the purposes of communication in the \#MND and \#ALS 'macro' structural layer of Twitter. [16]

Content analysis of the Twitter data involved hand-coding of excel spreadsheets by the first and third authors, to identify the numbers of tweets in each of the Dann [17] tweet content categories, for: (i) conversational tweets (mentioning another user), (ii) news (announcement and journalism), (iii) passalong tweets (sharing links to other Internet content), (iv) social presence tweets (showing connection with other Twitter users), and (v) status broadcast tweets, reflecting Twitter's use as a 'soapbox' where users communicate their thoughts, feelings, experiences, and 'diary of a daily life' content. The first and third author separately coded tweets according to these content classifications with differences of opinion on codes resolved by consensus. A qualitative analysis of the content themes of each written tweet in the Conversational and Status Broadcast tweets, that did not include links to other web content, was later performed in NVivo [18]. The Gephi and KH Coder visualisations are two complementary forms for representing different aspects of the Twitter data. Gephi networks show the interconnections between the individual participants, while KH Coder is used to provide a visual summary of the content of the tweet text corpus.

\subsection{Gephi visualisation method}

The NVivo program [15] was used to convert the captured Twitter data into Microsoft Excel [19] spreadsheets. The spreadsheet Twitter data were exported in comma separated values (CSV) format, and then imported into the Gephi program [20] to visualise the communication network embodied in the data. In the 3,288 tweets examined in this study, there were 2,665 unique nodes/users in the network produced from the data set, and 3,309 connection paths (of a least one tweet - directed or mention) between the nodes.

One approach to interpretation of social media data is social network analysis. The network data inherently created by social media activity represent the connections between individual participants as they interact, and can be used to make visible the 
social processes, and to identify prominent components and participants in the social network. As outlined in Figure 1 below, Gephi can be used to represent Twitter user accounts as 'nodes', and the communication path (representing one or more tweets) between two nodes as an 'edge'. In the Twitter network diagrams used in this paper, edges are presented as curved lines, the direction of tweets is clockwise around the edge, and the width of an edge is proportional to the total number of tweets recorded between the two nodes in that direction.

While there is a single topological arrangement of the data for a given network, it can be visualised in many ways. The Gephi program provides a range of algorithms for laying out networks. The Fruchterman-Reingold (F-R) layout algorithm [21] has a number of desirable characteristics (good node distribution, minimisation of edge crossings, uniform edge lengths, reflection of inherent symmetries, etc.), and was chosen for use here.

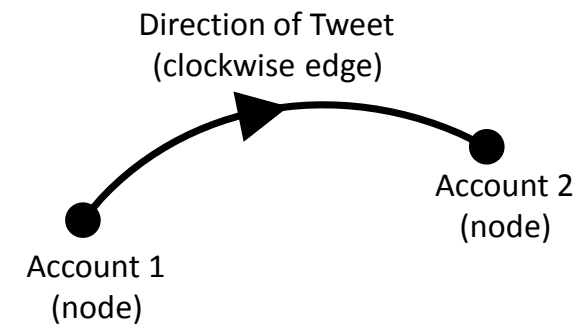

Figure 1. Twitter network visualisation schema used in this paper.

\subsection{KH Coder visualisation method}

The text analytics software package $\mathrm{KH}$ Coder [22] was used to analyse the text content of tweets collected. KH Coder was selected as it is free and provides a range of analysis and visualisation options. KH Coder supports the use of a dictionary of 'stop words', that is, words to be ignored in any analysis of the text [23]. Common English words and parts of speech, such as 'I', 'a', 'am', 'be', 'my', 'the', etc., add little to the analysis, and their relatively high frequency often masks the words/terms that are actually of significance [24]. A stop word dictionary was developed based on the example English stop word dictionary supplied with $\mathrm{KH}$ Coder, after inspection to remove any words likely to be relevant in the context here. A second issue that can mask the significance of words/terms in text analytics is the presence of inflected and/or derived forms of words, for example, a root word such as 'write' may also be present in the source text as 'writing', 'wrote', 'written', etc. KH Coder implements 'stemming' to consolidate inflected and derived words into their root form. Stemming via lemmatisation [24] based on English parts of speech (e.g., nouns, proper nouns, adjectives, verbs) was used. KH Coder supports a range of text data analysis and visualisation methods, we use here the co-occurrence network (CON) and multi-dimensional scaling (MDS) plot [25].

Co-occurrence refers to the presence of two (or more) terms in the same text unit of analysis - here we are interested if the same term groups frequently co-occur in individual tweets. $\mathrm{KH}$ Coder uses the Jaccard distance [23] as a measure of co-occurrence for term pairs. Based on specifying the minimum frequency of occurrence of a term for inclusion in the CON analysis and visualisation, terms appear as nodes in a network plot based on the F-R layout algorithm [21]. Frequently co-occurring terms in the visualisation are connected by lines/edges. It is possible to configure the plot to indicate the relative frequency of terms by the relative size of their node, and to indicate the relative frequency of cooccurrence of terms by the relative thickness of the edge connecting their nodes.

MDS computes a measure of 'distance' between all pairs of text terms, then seeks a representation of the terms in the least possible number of dimensions, such that original distance values between all term pairs are shown with the least error possible. Here we use two dimensions to produce a figure that is easy to display and interpret, acknowledging that there will necessarily be some error in the relative location of the text terms. KH Coder supports a number of distance measures and dimensional reduction techniques - here we use the Cosine distance measure [26] in combination with the Sammon method for dimensional reduction [27] in one case, and the Euclidean distance measure [28] in combination with the classical method for dimensional reduction [29] in the other case. Based on specifying the minimum frequency of occurrence of a term for inclusion in the MDS analysis and visualisation, terms appear as circles/bubbles in the plot, and it is possible to configure the plot to indicate the relative frequency of terms by the relative size of their bubble. Words/terms clustered close together in the resultant MDS visualisation are found more frequently close together in the source text, and may reveal key themes in the tweet data.

\subsection{Content category classification method}

A Twitter Content Classification system developed by Dann [17] was used to identify (i) 
conversational tweets (those mentioning another user), (ii) news (announcement and journalism), (iii) pass-along (sharing links to other Internet content, including quoted tweets as annotated media, and curation of other Internet content), (iv) social presence (with a Twitter audience), and (v) status broadcast, reflecting Twitter's use for communicating thoughts, feelings, experiences, and 'diary of a daily life' content. The first author and a research assistant separately coded tweets according to these content classifications with differences of opinion on codes resolved by consensus. The Conversational and Status Broadcast tweets became the focus for further analysis in the thematic content coding method.

\subsection{Thematic content coding method}

The first and third authors had read and re-read the tweets for aforementioned coding purposes, and moved on to a reading of each tweet coded as 'Conversational' and 'Status Broadcast' tweets. These tweets were imported into NVivo [18] for qualitative coding of content according to content themes. Open coding involved reading each tweet and considering the context of the hashtags (e.g., \#MNDwidow, \#dedicated, \#fighter, \#MNDproblems, \#BeatALS) and applying a code as a unit of meaning reflected in the tweet (e.g., sympathy; living with MND/ALS; humour). The codes were then grouped according to categories, and connecting themes identified by matrix coding - looking for relationships between the codes. The first and third authors discussed the open codes and categories of meaning to arrive at the final content themes.

\section{Results}

Excel was used to calculate the total number of tweets with ALS and/or MND in the tweets, to triangulate with $\mathrm{KH}$ Coder results showing little cooccurrence of MND and ALS in the data. Results presented in Figure 2 support the analysis in the $\mathrm{KH}$ Coder CON graphic. That 358 were tagged with both, however, indicates that at least in Twitter the tags might be viewed as interchangeable for the purposes of communicating about either MND or ALS.

\subsection{Gephi analysis visualisation}

For the period under investigation a total of 3288 tweets were included in the sample. These originated from 1574 unique Twitter user accounts, and connected 2655 unique user accounts (nodes) via 3309 unique pathways (edges). There were 2785 undirected tweets (i.e., those that did not have an @ symbol at the front) although these may have mentioned other users. As such, a tweet in the graphic may show more than one edge. Points of interest are labelled and discussed further below. Based on the schema presented in Figure 1 and using the F-R layout algorithm, Figure 3 presents a visualisation of the Twitter network data included in the study.

Without retweets, the network density (crosslinking between nodes) in this sample is low, so even though there are a high number of nodes $(n=2665)$ and edges $(n=3309)$, the network has a distinct 'star' shape. That is, several tweeters are sending their tweets to no one in particular, generating the whirlpool surrounding the large 'undirected' node. Some 'busy' tweeters can be seen by the wide edges travelling from them clockwise inward to 'undirected'. Toward the outer edge of the whirlpool are some people with clockwise outward connections to others - directed and/or mentioning tweets to other people - generally without a reply (a reply on the tag would otherwise appear as a matching edge in the opposite direction forming a loop between the two nodes).

Some of the MND/ALS organisational handles have a low number of outward bound tweets tagged with \#MND \#ALS in the sample period, but have been mentioned by other users quite frequently; their nodes are sitting at the centre of clockwiseinward mini-spirals. Around the very outside are directed tweets - generally someone at the outer edge sending a directed tweet to one or a small number of other users. The labels on the Gephi represent nodes featuring in the network with more tweets or the subject of tweets. P2 (a person with MND/ALS) made the most tweets/mentions; P3 tweeted to/mentioned the most unique other users; F3 (family member) made the most undirected tweets; O1 was most tweeted/mentioned - followed by $\mathrm{O} 2$ (support organisations).

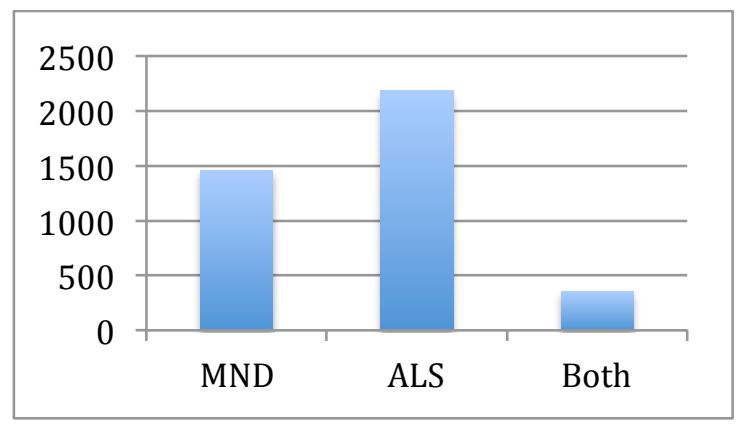

Figure 2. Frequency of MND and ALS on tweets 


\subsection{KH Coder (MDS, CON) visualisations}

The KH Coder MDS and CON visualisations of the \#ALS and \#MND shows several evocative concepts (shown in Figure 4, Figure 5, and Figure 6) including 'life/die' 'fight/battle/lose' 'family' 'cure' 'thanks/love' and 'patient/story'. The concept clusters (Figure 5) are: (i) MND/ALS as a health condition, associated with impairments and disability, and associated with support organisations; (ii) BMAA and purported links with other neurodegenerative conditions; (iii) raising funds and charity; (iv) raising awareness; (v) activity of support associations, and (vi) the death of 'Kevin Turner' (NFL player).

\subsection{Content classifications of tweets}

Conversational tweets are those that contain a @ user handle within the tweet. These include conversational replies (an @user reply) conversational referrals (e.g., referring one user to another in the same tweet, or referring a user to a link provided with the tweet), and referring to the @user as being present (rhetorical presence) [17]. The frequencies of tweets in content categories are displayed in Figure 7 below. The data set contained a preponderance of tweets containing links, present in both conversational and pass along tweets on the tags.

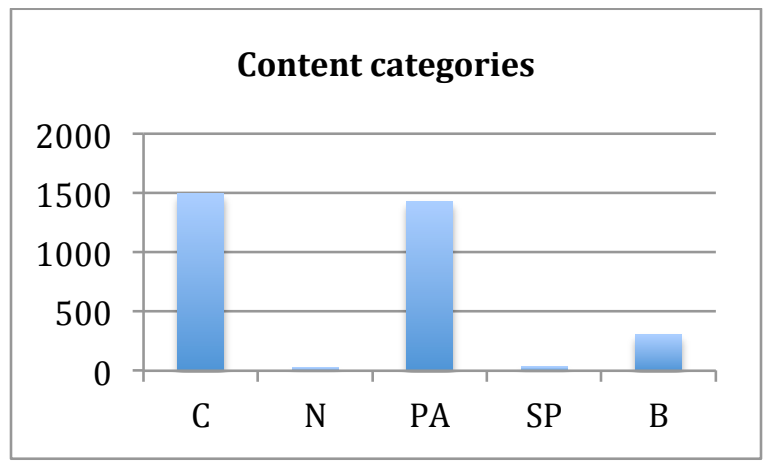

Figure 7. Frequency of content categories of tweets Key: $\mathrm{C}=$ conversational; $\mathrm{N}=$ news; $\mathrm{PA}=$ pass along; $\mathrm{SP}=$ social presence; $\mathrm{B}=$ status broadcast.

\subsection{Content thematic analysis}

Conversational and Status Broadcast tweets were a rich source of patient, family, and general public thoughts and sentiment in relation to MND/ALS. Overall, the content of Conversational and Status Broadcast tweets reflected Twitter being used to express a 'quest for a cure' and 'quest for a better life' along the 'devastating' path of living with MND/ALS, and use of Twitter to: (i) converse or share opinion on science, research, evidence, and guidance; (ii) deliver insights on living with MND/ALS; (iii) engage directly with public figures and the media to lobby government and peak organisations, to improve services and funding for people with MND/ALS; (iv) offer personal words of encouragement, support, help, and thanks for people and organisations supporting fundraising efforts, and (v) provide positive, uplifting, supportive and encouraging words to people with ALS and their families (a fighter, an inspiration, brave, loved, supported) and personal messages of condolence (e.g., in response to the deaths of Ronnie Corbett; Kevin Turner) including offers of 'doing anything' to help the person.

The major categories (also evident in Figure $4 \mathrm{KH}$ Coder MDS plot) were linked thematically by several common themes in tweets, including: (i) MND/ALS having no known cause or cure, and being a devastating disease (cruel, awful, nothing worse, a higher priority than other conditions); (ii) MND/ALS affecting family members, friends, and others, who expressed sorrow, support, and empathy for people with MND/ALS or spouses as they together 'fight' or 'battle' the disease; (iii) people with MND/ALS needing not only aids and equipment, but an accessible environment and a knowledgeable society that is aware of the disease and its impacts; (iv) the use of dark humour and irony in tweets, at loss of both an 'old life' (reflecting on the person as they were before MND/ALS) and of an imagined future life. Twitter was also used to share insights on endof-life decisions, and dying with dignity. Bereaved family members continued to use Twitter to voice the need for support after the death of the person with MND /ALS. 


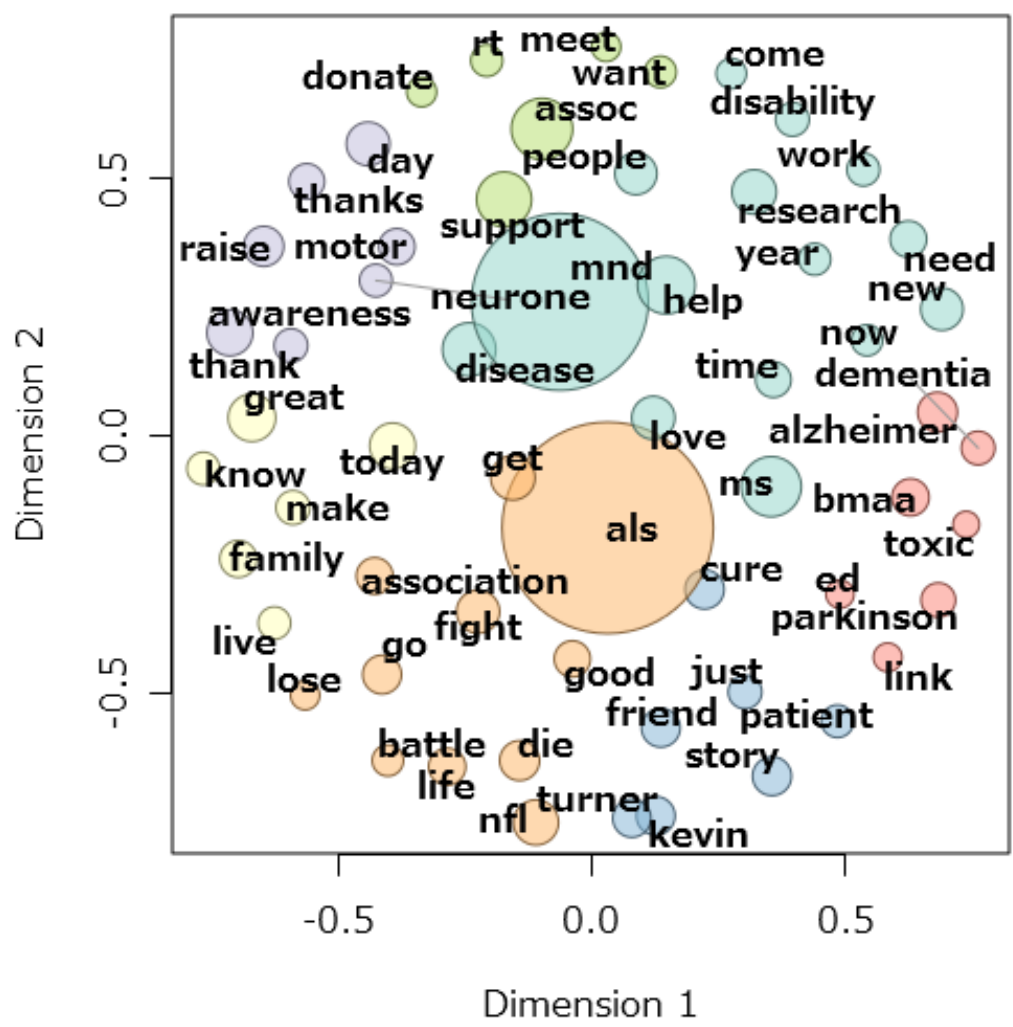

Figure 4. KH Coder MDS plot - Cosine distance, Sammon method

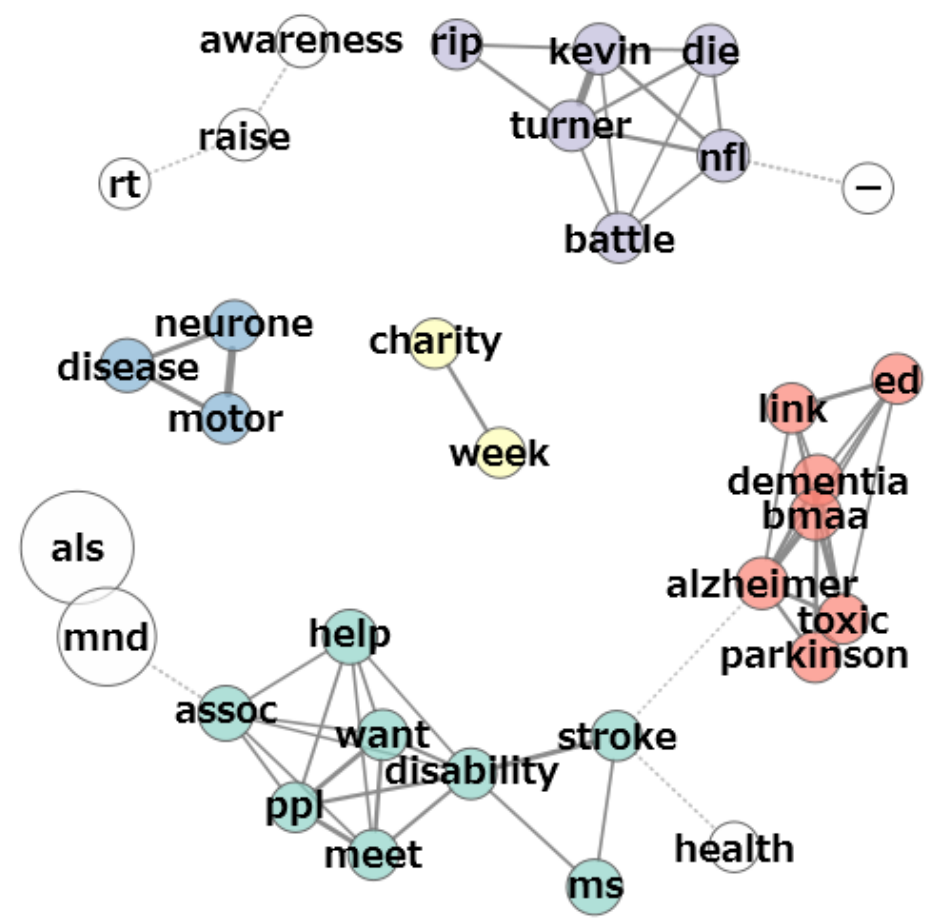

Figure 5. KH Coder - Co-Occurrence Network 


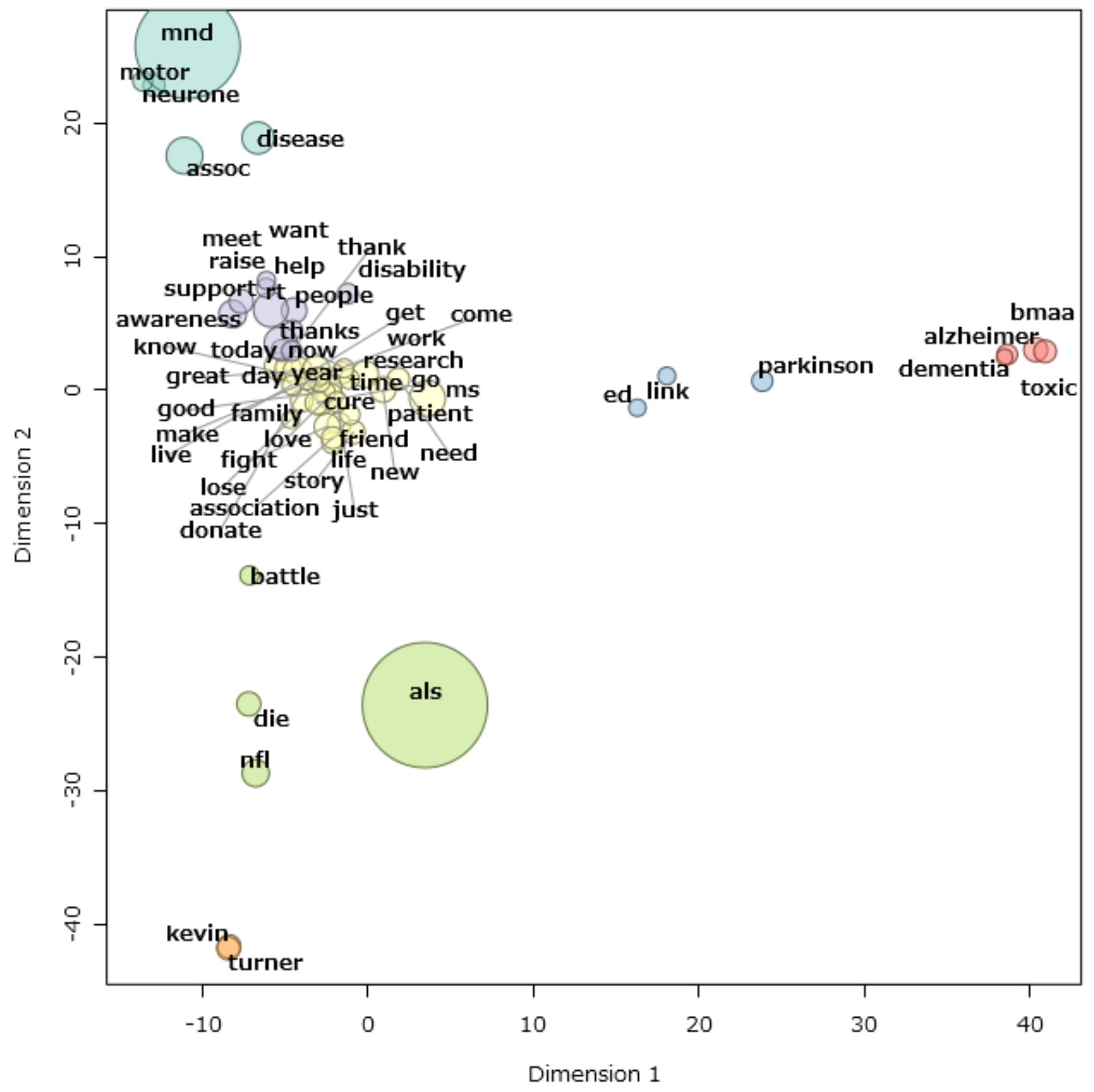

Figure 6. MDS Plot - Euclidean distance, Classical method 


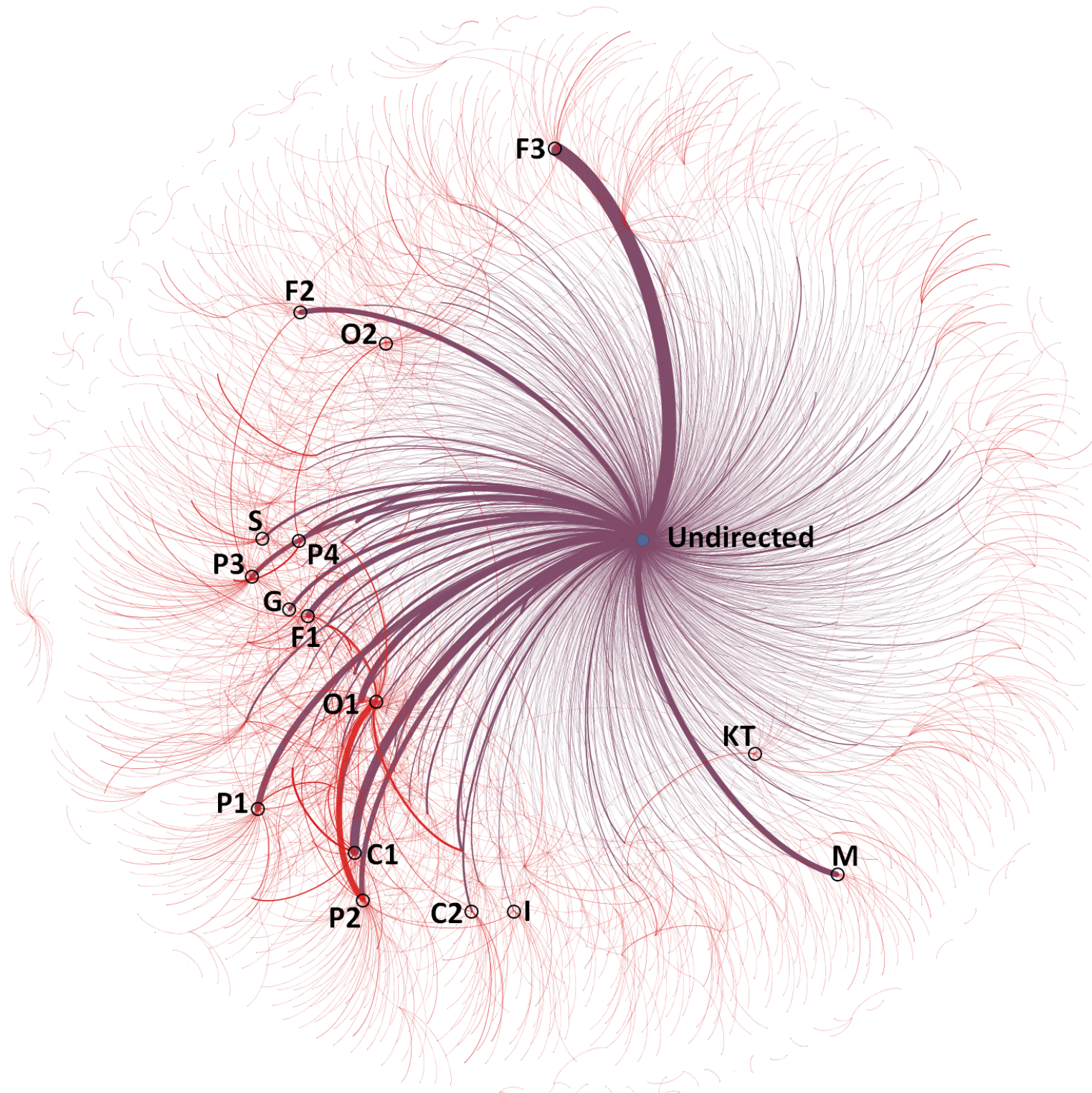

Figure 3. Gephi visualisation

In Figure 3 above, nodes are labelled to show nodes with more activity in the network, and their relationship with one another and the undirected node as labelled: $\mathrm{C}=$ campaigner for $\mathrm{MND} / \mathrm{ALS}$ organisation, $\mathrm{F}=$ Family, $\mathrm{G}=$ product (goods and services), $\mathrm{I}=$ individual (not classified), $\mathrm{KT}=$ Kevin Turner, $\mathrm{M}=$ media, $\mathrm{O}=$ organisation for $\mathrm{MND} / \mathrm{ALS}$, $\mathrm{P}=$ person with $\mathrm{MND} / \mathrm{ALS}, \mathrm{S}=$ Celebrity. People with MND/ALS and their families are connecting organisations with a wider audience. The top $5 \%$ of tweeters generated more than a third of all tweets, and only $15 \%$ of tweets were directed. This reflects use of the platform as a 'megaphone' and by a relatively small number of influential tweeters, whose support on messages could be harnessed to amplify a variety of health messages on MND/ALS in Twitter.

\section{Discussion}

The results of this study show that people with MND/ALS and their families are using Twitter for the purposes of interpersonal communication, information exchange, and social closeness, particularly in relation to the experience of living with MND/ALS, and the need for support and 
resources. The preponderance of Conversational and Pass Along tweets containing links in the dataset did not impede analysis of the content of tweets reflecting the Twitter users' own perspectives or views, albeit to a lesser extent, by relatively few tweets of the Status Broadcast type on the hashtag during the data collection period. In terms of methodological, theoretical and practical implications, the mixed methodologies permitted (a) new insights into the large dataset with the computational methods augmenting and verifying the information gathered through a separate qualitative analysis, and (b) an indicator of the relative proximity of concepts according to their frequency of appearance within the dataset, reflecting a distillation of the overall 'conversation' occurring in the network on \#MND and \#ALS. This allowed the 'voices' of the people with MND/ALS, and their families and service providers, to come through in the results with greater clarity. In the context of the study being limited to the macro structural layer of Twitter, the tagged tweets were meant for a broader audience [16] than the tweeters' own followers, in effect a 'public' expression of the themes presented in the results. That people with MND/ALS and their families are motivated to tweet to the tags \#MND and/or \#ALS, including tweets directed to politicians to lobby for improved funding and policy support, and to share their own views on research priorities, suggests that their use of Twitter goes beyond conversational interaction and information exchange, to comprise a form of political action. For a group with severe communication disability, Twitter may offer a unique mode of direct and publication communication to adults with MND/ALS who have access to the Internet and use social media. The content results relating to research are also evidence of engagement in 'research translation' and the need for Twitter content to include curation of scientific information about MND/ALS of high interest, relevance, and importance to people with these conditions.

\section{Directions for Future Research}

This hashtag study, with purposively selected tweets, revealed that much goes on in Twitter in relation to MND/ALS, beyond its use for fundraising or lobbying for awareness. However, it is limited by not determining the proportion of tweets in the sample by people with MND/ALS or their families [16], and was limited to the macro layer of Twitter. As such, it is not known whether these patterns of use and content themes are similar across layers [16] or related to the impacts or stages of MNS/ALS. Further research is needed to include large samples of tweets from people with MND and their families, harvested from the profiles with informed consent. Such data would further develop the content themes presented in this study and lead to improved understanding of how tweeters with MND/ALS and their families are approaching the platform for multiple purposes of engagement. Interviews with people with MND/ALS, either by email or short interviews, or observations of Twitter use in naturalistic settings, would enable exploration of the use of assistive or adaptive technologies, augmentative and alternative communication methods and/or a scribe to write tweets, particularly in the end stages of the disease.

The clinical and training implications arising from this research are that people interested to hear the views of many people with MND/ALS might consider learning to use Twitter as an important 'social media' channel for hearing the lived experiences of people with MND and their families. That tweeters in this study intended their messages for the macro structural layer of Twitter, shows that they wish a wider public audience to take heed of their '\#MNDproblems' and to be more aware and alert to what it means to live with and 'battle' MND/ALS.

\section{References}

[1] S. A. Moorehead, D. E. Hazlett, L. Harrison, J. K. Carroll, A. Irwin, and C. Hoving, "A New Dimension of Health Care: Systematic Review of the Uses, Benefits, and Limitations of Social Media for Health Communication", Journal of Medical Internet Research, 2013, e85.

[2] B. Hemsley, S. Palmer, and S. Balandin, "Tweet Reach: A Research Protocol for Using Twitter to Increase Information Exchange in People with Communication Disabilities." Developmental Neurorehabilitation, United States, Taylor and Francis, 2014, pp. 84-89.

[3] B. Hemsley, S. Dann, S. Palmer, and S. Balandin, "We Definitely Need an Audience": Experiences of Twitter, Twitter Networks and Tweet Content in Adults with Severe Communication Disabilities who use Augmentative and Alternative Communication (AAC)", Disability and Rehabilitation, United Kingdom, Taylor and Francis, 2015, pp. 1531-1542.

[4] M. Brunner, B. Hemsley, S. Palmer, S. Dann, and L. Togher, "Review of the Literature on the Use of Social Media by People with Traumatic Brain Injury (TBI)", Disability and Rehabilitation, United Kingdom, 2015, 1511-1521.

[5] H. Paterson, and C. Carpenter, "Using Different Methods to Communicate: How Adults with Severe 
Acquired Communication Difficulties Make Decisions about the Communication Methods they Use and How They Experience Them", Disability and Rehabilitation, United Kingdom, 2015, 1522-1530.

[6] B. Hemsley, and S. Palmer, "Two Studies on Twitter Networks and Tweet Content in Relation to Amyotrophic Lateral Sclerosis (ALS): Conversation, Information, and Diary of a Daily Life", Studies in Health Technology and Informatics, 2016, 227, 41-47.

[7] Access Economics. "Economic Analysis of Motor Neurone Disease in Australia." Deloitte, Australia, November 2015.

[8] National Institute for Health and Clinical Excellence, "Motor Neurone Disease: The Use of Non-Invasive Ventilation in the Management of Motor Neurone Disease, NICE Clinical Guideline 105" National Institute for Health and Clinical Excellence, London, 2010.

[9] Anderson, P. M., S. Abrahams, G. D. Borasio, et al, "EFNS Guidelines on the Clinical Management of Amyotrophic Lateral Sclerosis (MALS) - Revised Report of an EFNS Task Force", European Journal of Neurology, 2012, 19(3), pp. 360-376.

[10] R. G. Miller, B. R. Brooks, R. J. Swain-Eng, et al, "Quality Improvement in Neurology: Amyotrophic Lateral Sclerosis Quality Measures: Report of the Quality Measurement and Reporting Subcommittee of the American Academy of Neurology", Neurology, 2013, 81(24), pp. 2136-2140.

[11] A. G. Chio, G. Logroscino, B. J. Traynor, J. Collins, J. C. Simeone, L. A. Goldstein, and L. A. White, "Global epidemiology of amyotrophic lateral sclerosis: a systematic review of the published literature." Neuroepidemiology, 2013, p.118-130.

[12] Beukelman, D. R., and P. Mirenda. "Augmentative and Alternative Communication: Supporting Children and Adults with Complex Communication Needs, Fourth Edition”, Brookes Publishing, Baltimore, 2013.

[13] Beukelman, D., S. Fager, and A. Nordness, "Communication Support for People with ALS", Neurology Research International, 2011, Article ID 714693, 6 pages (online).

[14] N. M. Phing, and R. Yazdanifard. "How does ALS Ice Bucket Challenge Achieve its Viral Outcome through Marketing via Social Media?" Global Journal of Management and Business. 17(7-E).

[15] QSR International. (2012). NCapture (Version 1.0.72.0). Doncaster, Victoria: QSR International.

[16] Bruns, A. and H. Moe, "Structural Layers of Communication in Twitter. In: Weller, K., Bruns, A.,
Burgess Mahrt M, Puschmann, C. Eds. Twitter and Society. New York: Peter Lang; 2014, pp. 15-28.

[17] Dann, S. "Benchmarking Microblog Performance: Twitter Content Classification Framework". In Burkhalter, J. N., Wood, N. T. Eds. Maximizing Commerce and Marketing Strategies through Micro-Blogging, 2015, Hershey, PA: IGI Global.

[18] QSR International "NVivo Version 10.0.138.0". Doncaster, Victoria. 2012, QSR International.

[19] Microsoft. "Excel Version 14.0.6112.5000". Redmond, Washington, Microsoft, 2010.

[20] The Gephi Consortium. "Gephi Version 0.8.1", The Gephi Consortium, Paris, 2012

[21] T. M .J. Fruchterman, and E. M. Reingold, E. "Graph drawing by force-directed placement." Software: Practice and Experience, 1991, 21(11), 1129-1164.

[22] Higuchi, K. (2014). KH Coder (Version 2.00beta.32). Japan: Koichi Higuchi. Retrieved from http://khc.sourceforge.net/en/

[23] Hu, X. and H. Liu, "Text Analytics in Social Media." In C. C. Aggarwal \& C. Zhai (Eds.), Mining Text Data (pp. 385-414): Springer US. 2012.

[24] R. Bolden, and J. Moscarola. "Bridging the Quantitative-Qualitative Divide: The Lexical Approach to Textual Data Analysis." Social Science Computer Review, 2000, 18(4), 450-460.

[25] Namey, E., G. Guest, L, Thairu, and L. Johnson, L. "Data reduction techniques for large qualitative data sets." In G. Guest \& K. M. MacQueen (Eds.), Handbook for team-based qualitative research, Plymouth, UK: Altamira Press, 2007, pp. 137-162.

[26] Jing, L., Zhou, L., Ng, M. K. \& Huang, J. Z. (2006). "Ontology-based Distance Measure for Text Clustering." Proceedings of the SIAM SDM workshop on text mining, Bethesda, Maryland

[27] Sammon, J. W., "A Nonlinear Mapping for Data Structure Analysis." IEEE Transactions on computers, 1969, C-18(5), 401-409.

[28] T. Yamada, K. Yamashita, N. Ishii, and K. Iwata, K. "Text Classification by Combining Different Distance Functions with Weights." Proceedings of the Seventh ACIS International Conference on Software Engineering, Artificial Intelligence, Networking, and Parallel/Distributed Computing, Las Vegas, 2006, 19-20 June, pp. 85-90.

[29] H. Abdi, "Metric Multidimensional Scaling. Encyclopedia of Measurement and Statistics", Thousand Oaks, CA: Sage Publications, Inc In N. J. Salkind \& K. Rasmussen (Eds.) 2007, pp. 599-606. 